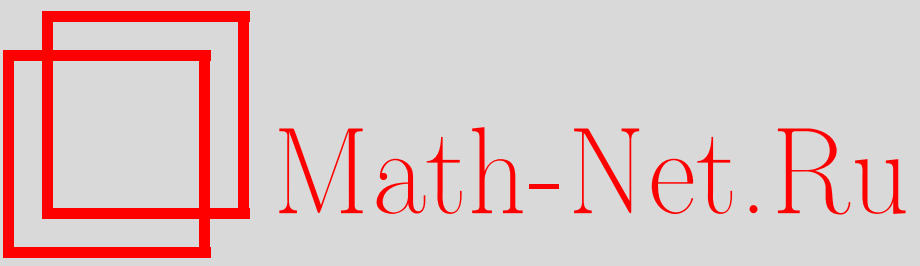

В. В. Абрамов, Математическое моделирование тесных сближений малых тел солнечной системы с большими планетами и Луной, Вестн. Сам. гос. техн. ун-та. Сер. Физ.-мат. науки, 2007, выпуск 2(), 151-154

DOI: https://doi.org/10.14498/vsgtu545

Использование Общероссийского математического портала Math-Net.Ru подразумевает, что вы прочитали и согласны с пользовательским соглашением http://www. mathnet.ru/rus/agreement

Параметры загрузки:

IP : 54.164 .48 .24

26 апреля 2023 г., $18: 37: 24$ 
Вестн. Сам. гос. техн. ун-та. Сер.: Физ.-мат. науки. - 2007. - № 2(15). - С. 151-154. - ISSN 1991-8615

УдК 521.1; 523.642

В. В. Абрамов

\section{МАТЕМАТИЧЕСКОЕ МОДЕЛИРОВАНИЕ ТЕСНЫХ СБЛИЖЕНИЙ МАЛЫХ ТЕЛ СОЛНЕЧНОЙ СИСТЕМЫ С БОЛЬШИМИ ПЛАНЕТАМИ И ЛУНОЙ}

Обоснован выбор метода численного интегрирования уравнений движения малых тел Солнечной системъ в моменты их тесных сближений с большими планетами или Луной. Проведённое исследование показало, что в такие моменты более предпочтительным является применение многошагового метода АдамсаМултона с заранее выбранным менъшим шагом и увеличенным порлдком аппроксимачии. Моделирование моментов сближений с помощью одношагового метода Эверхарта с переменным шагом значительно увеличивает общее время вычислительного прочесса и требует дальнейшей оптимизачии критерия изленения uara.

Основной проблемой при математическом моделировании движения малых тел Солнечной системы является сохранение устойчивости решения дифференциальных уравнений движения в случае наличия тесных сближений малых тел с большими планетами или Луной. Это связано с тем, что в момент тесного сближения с планетой у малого тела за короткий промежуток времени происходит значительное изменение компонентов вектора ускорения. Приём, величина этого промежутка обычно меньше временной величины общего шага интегрирования и составляет от нескольких часов при умеренно тесных сближениях до нескольких десятков минут при особо тесных сближениях, тогда как общий шаг интегрирования обычно измеряется десятками часов. Следствием этого является нарушение гладкости кривых изменения координат и компонентов вектора скорости.

Наиболее чувствительными к подобного рода скачкам являются разностные многошаговые методы, например, методы Адамса [1]. Если не уменьшать шаг интегрирования в моменты тесных сближений, то происходит потеря устойчивости решения, что приводит к сильному искажению результата.

Поскольку моменты тесных сближений являются кратковременными по сравнению с общей продолжительностью вычислительного процесса, применение методов Адамса является вполне оправданным для численного интегрирования уравнений движения малых тел при отсутствии тесных сближений. Это связано с высокой эффрективностью многошаговых методов. Например, с помощью неявного метода Адамса-Мултона 11-го порядка можно добиться практически такой же точности, что и в модифицированном методе Эверхарта 27-го порядка. При отсутствии тесных сближений небесных тел друг с другом процесс численного интегрирования методом Адамса-Мултона с шагом 0,2 дня происходит в 4-5 раз быстрее, чем методом Эверхарта с шагом в 1 день [2]. Поэтому метод Адамса-Мултона был выбран для решения уравнений движения малых тел в отсутствие сближений.

Данный многошаговый метод является неявным, следовательно, при его использовании необходимо решать нелинейное уравнение, например, с помощью итерационного процесса [1]:

$$
\begin{aligned}
& P: y_{n+1}^{(0)}=y_{n}+h \sum_{i=0}^{k} B_{i} f\left(x_{n-i}, y_{n-i}\right), \\
& E: f_{n+1}^{(1)}=f\left(x_{n+1}, y_{n+1}^{(0)}\right), \\
& C: y_{n+1}^{(1)}=y_{n}+h M_{0} f_{n+1}^{(1)}+h \sum_{i=1}^{k} M_{i} f\left(x_{n+1-i}, y_{n+1-i}\right), \\
& \quad \vdots \\
& E: f_{n+1}^{(v)}=f\left(x_{n+1}, y_{n+1}^{(v-1)}\right), \\
& C: y_{n+1}^{(v)}=y_{n}+h M_{0} f_{n+1}^{(v)}+h \sum_{i=1}^{k} M_{i} f\left(x_{n+1-i}, y_{n+1-i}\right)=y_{n+1}, \\
& E: f_{n+1}=f\left(x_{n+1}, y_{n+1}\right) .
\end{aligned}
$$

Процесс вычислений (1) называется методом прогноза и коррекции $P(E C)^{v} E$. Запись $P$ означает применение предсказывающей формулы метода Адамса-Бэшфорта, $E$ - вычисление правой части дифференциального уравнения, $C$ - применение исправляющей формулы метода Адамса-Мултона; $v$-количество итераций, $B_{i}$ и $M_{i}$-коэфффициенты метода Адамса-Бэшфорта 
и метода Адамса-Мултона соответственно. Таким образом, сначала производится вычисление начального приближения $y_{n+1}^{(0)}$ по формуле метода Адамса-Бэшфорта, которое затем используется в вычислении правой части дифференциального уравнения - функции $f$, входящей в формулу метода Адамса-Мултона. Итерации продолжаются до тех пор, пока не будет достигнута сходимость, либо фриксированное число раз.

Если продолжать численное интегрирование с помощью метода Адамса-Мултона в моменты тесных сближений, то необходимо увеличить порядок аппроксимации с 11 до 16, а также уменьшить шаг интегрирования [3].

Проблему сохранения устойчивости также можно решить путём применения в моменты тесных сближений метода Эверхарта [4], который обладает большей устойчивостью по сравнению с многошаговыми методами. Эфрфективность данного неявного одношагового метода была подтверждена путём сопоставления результатов вычислений координат и скоростей небесных тел с базой данных DE 405, которая в свою очередь согласована с радиолокационными наблюдениями [5]. Другим преимуществом метода Эверхарта является простота смены шага интегрирования. То есть данный метод, как и любой другой одношаговый, позволяет плавно изменять шаг, например, уменьшать его при увеличении модуля ускорения малого тела.

Для сравнения эффрективности методов Адамса и Эверхарта, они применялись к одним и тем же дифференциальным уравнениям движения, описывающим математическую модель движения небесных тел. При вычислениях, помимо взаимного влияния небесных тел, учитывались релятивистские эффректы. Для этого необходимо было использовать дифференциальные уравнения движения в барицентрической системе координат с учётом ньютоновских и шварцшильдовских членов [6]:

$$
\begin{aligned}
\ddot{\boldsymbol{r}}_{i}=k^{2} \sum_{j \neq i} m_{j} \frac{\boldsymbol{r}_{j}-\boldsymbol{r}_{i}}{r_{i j}^{3}}\left(1-\frac{2 k^{2}(\beta+\gamma)}{c^{2}} \sum_{k \neq i} \frac{m_{k}}{r_{i k}}-\frac{k^{2}(2 \beta-1)}{c^{2}} \sum_{k \neq j} \frac{m_{k}}{r_{j k}}+\right. \\
\left.+\gamma\left(\frac{v_{i}}{c}\right)^{2}+(1+\gamma)\left(\frac{v_{j}}{c}\right)^{2}-\frac{2(1+\gamma)}{c^{2}} \dot{\boldsymbol{r}}_{i} \dot{\boldsymbol{r}}_{j}-\frac{3}{2 c^{2}}\left(\frac{\left(\boldsymbol{r}_{i}-\boldsymbol{r}_{j}\right) \dot{\boldsymbol{r}}_{i}}{r_{i j}}\right)^{2}+\frac{1}{2 c^{2}}\left(\boldsymbol{r}_{j}-\boldsymbol{r}_{i}\right) \ddot{\boldsymbol{r}}_{j}\right)+ \\
\quad+\frac{k^{2}}{c^{2}} \sum_{j \neq i} \frac{m_{j}}{r_{i j}^{3}}\left(\left(\boldsymbol{r}_{i}-\boldsymbol{r}_{j}\right) \times\left((2+2 \gamma) \dot{\boldsymbol{r}}_{i}-(1+2 \gamma) \dot{\boldsymbol{r}}_{j}\right)\right)\left(\dot{\boldsymbol{r}}_{i}-\dot{\boldsymbol{r}}_{j}\right)+\frac{k^{2}(3+4 \gamma)}{2 c^{2}} \sum_{j \neq i} \frac{m_{j} \ddot{\boldsymbol{r}}_{j}}{r_{i j}} .
\end{aligned}
$$

Здесь $\boldsymbol{r}_{i}, \dot{\boldsymbol{r}}_{i}, \ddot{\boldsymbol{r}}_{i}$ - координаты, скорость, ускорение в барицентрической системе координат $i$-го возмущаемого тела; $\boldsymbol{r}_{j}, \dot{\boldsymbol{r}}_{j}, \ddot{\boldsymbol{r}}_{j}$ - координаты, скорость, ускорение в барицентрической системе координат $j$-го возмущающего тела; $k^{2}$-гравитационная постоянная, $m_{j}$-масса $j$-го тела; $r_{i j}=\left|r_{j}-r_{i}\right|, v_{i}=\left|\dot{\boldsymbol{r}}_{i}\right| ; \beta$ и $\gamma$ - релятивистские параметры $(\beta=\gamma=1) ; c-$ скорость света.

В настоящей работе большое внимание уделено проблеме выбора шага интегрирования и критерия начала сближения. Как уже было отмечено выше, в моменты тесных сближений можно либо продолжать численное интегрирование одношаговым методом Эверхарта с переменным шагом, либо многошаговым методом Адамса с постоянным, но меньшим шагом.

Для первого случая был выбран следующий алгоритм определения шага интегрирования:

$$
h(a)=\left\{\begin{array}{lll}
h_{0}, & a \leqslant a_{0} & \text { (метод Адамса), } \\
h_{0} \sqrt{\frac{a_{0}}{a}}, & a>a_{0} & \text { (метод Эверхарта), }
\end{array}\right.
$$

где

$$
a_{0}=k^{2} \frac{m_{0}}{r_{0}^{2}}
$$

Величина шага интегрирования $h$ зависит только от величины $a$, которую, например, можно взять равной

$$
a=\max _{i=1,2, \ldots, n} a_{i}
$$

где $a_{i}$ - модуль ускорения, создаваемого $i$-м телом, влияющим на $j$-е тело, движение которого исследуется $(i \neq j)$. При этом Солнце в множество объектов $a_{i}$ не входит.

Все остальные величины считаются известными ещё до запуска алгоритма и определяются опытным путём. Пусть известно, что при интегрировании с шагом $h_{0}$ минимально-возможное 
расстояние между исследуемым телом и некоторым телом массой $m_{0}$, при котором не нарушается устойчивость, равно $r_{0}$. Таким образом, величина $a_{0}$ является «критическим» ускорением, при возникновении которого следует начать уменьшать шаг. Например, численное интегрирование методом Адамса-Мултона с шагом $h_{0}=0,2$ дня можно производить до тех пор, пока исследуемый объект не сблизится с Землёй (масса $m_{0}=3,00349 \cdot 10^{-6}$ кг) на расстояние $r_{0}=0,02$ a. е. Тогда $a_{0}=2,22192 \cdot 10^{-6}-$ согласно (4), критическое ускорение, при котором необходимо начать уменьшать шаг.

При возникновении максимального ускорения $a$, равного критическому $a_{0}$, сообщаемого одним из тел, за исключением Солнца, необходимо переключиться на метод Эверхарта и в дальнейшем считать с переменным шагом $h$, согласно второму условию фрормулы (3), до тех пор, пока ускорение $a$ снова не станет меньше критического $a_{0}$. В этот момент необходимо вновь продолжить интегрирование с помощью метода Адамса-Мултона.

Во втором случае, когда метод Адамса с постоянным шагом используется и в моменты тесных сближений (и при их отсутствии) фрормула (3) принимает вид

$$
h(a)= \begin{cases}h_{0}, & a \leqslant a_{0}, \\ \frac{h_{0}}{l}, & a>a_{0}, \quad l=\text { const. }\end{cases}
$$

Таким образом, в моменты тесных сближений величину шага интегрирования $h_{0}$ необходимо уменьшить в $l$ раз. Число $l$ также определяется опытным путём и является достаточно большим. Для очень тесных сближений шаг интегрирования следует уменьшать в пять и более тысяч раз.

Второй способ, несмотря на его простоту, показал более хорошие результаты. Данное обстоятельство было выявлено при исследовании сходимости решения уравнений движения астероида 99942 Apophis, имеющего несколько тесных сближений на интервале времени 200 лет, при использовании двух рассмотренных выше способов.

Для каждого способа численное интегрирование дифференциальных уравнений движения (2) осуществлялось с различным шагом. Закладка начальных данных для старта многошагового метода производилась с помощью метода Эверхарта. Для первого способа значение критического ускорения в фрормуле (3) было выбрано равным $a_{0}=2,22192 \cdot 10^{-6}$. Для второго способа в формуле (6) величина $l=5000$.

Сначала численное интегрирование производилось с помощью первого способа с начальным шагом $h_{01}=0,2$. В результате вычислялись координаты $x_{1}(t), y_{1}(t), z_{1}(t)$ астероида Арорһis для некоторых моментов времени $t$. Затем интегрирование проводилось заново с другим начальным шагом $h_{02}=0,125$. В результате определялись координаты $x_{2}(t), y_{2}(t), z_{2}(t)$ несколько отличающиеся от координат $x_{1}(t), y_{1}(t), z_{1}(t)$ для тех же моментов времени. Для сравнения полученных координат вычислялись расстояния между положениями астероида, соответствующих координатам $x_{1}(t), y_{1}(t), z_{1}(t)$ и координатам $x_{2}(t), y_{2}(t), z_{2}(t)$, то есть определялись величины

$$
r(t)=\sqrt{\left(x_{1}(t)-x_{2}(t)\right)^{2}+\left(y_{1}(t)-y_{2}(t)\right)^{2}+\left(z_{1}(t)-z_{2}(t)\right)^{2}},
$$

по которым можно судить о точности вычислений. Аналогично определялись величины $r(t)$ по фороуле (7) и для второго способа.

В таблице для различных моментов времени представлены отклонения $r(t)$ в километрах, вычисленные по фрормуле (7), для астероида 99942 Арорhis на интервале интегрирования с 31 декабря 2006 года по 1 июня 2207 года при использовании в моменты тесных сближений либо метода Эверхарта, либо Адамса-Мултона. Как видно из таблицы, при использовании в моменты сближений метода Эверхарта сходимость решения после 2013 года становится хуже, чем при использовании метода Адамса-Мултона. Расхождения $r(t)$ во втором столбце становятся примерно на порядок больше, чем в третьем. Связано это с тем, что в 2013 году произойдет одно из сближений астероида 99942 Арорhis с Землей, не являющееся тесным [7].

Однако после тесного сближения данного астероида с нашей планетой 13 апреля 2029 года [8] дальнейшего ухудшения сходимости решения методом Эверхарта не происходит. Расхождения $r(t)$ для первого способа продолжают превышать расхождения $r(t)$ для второго способа до конца исследуемого временного интервала примерно на порядок.

На рисунке для астероида 99942 Apophis изображены графики логарифмов отклонений $r$ при шаге интегрирования 0,2 и 0,125 дня в зависимости от интервала интегрирования $t$, представленного в днях, при использовании в моменты тесных сближений либо метода Эверхарта, 
Отклонения $\boldsymbol{r}$ (км) при использовании различных методов в моменты тесных сближений при шаге 0,2 и 0,125 дня

\begin{tabular}{|c|c|c|}
\hline Дата & Метод Эверхарта & Метод Адамса \\
\hline 22.06 .2012 & 0,0000017 & 0,0000015 \\
27.07 .2013 & 0,0000080 & 0,0000028 \\
31.05 .2020 & 0,0000791 & 0,0000107 \\
17.09 .2026 & 0,0003519 & 0,0000369 \\
24.02 .2046 & 76,261015 & 6,5681913 \\
03.08 .2065 & 2,8712877 & 0,2488104 \\
10.01 .2085 & 102,50660 & 8,6936867 \\
20.06 .2104 & 24,886484 & 2,1058441 \\
28.11 .2123 & 100,97956 & 8,5690589 \\
07.05 .2143 & 851,69127 & 72,216922 \\
27.04 .2160 & 2056,1727 & 174,80432 \\
05.10 .2179 & 4899,2355 & 415,65118 \\
14.03 .2199 & 78420,181 & 6705,8011 \\
01.06 .2207 & 127471,42 & 12048,359 \\
\hline
\end{tabular}

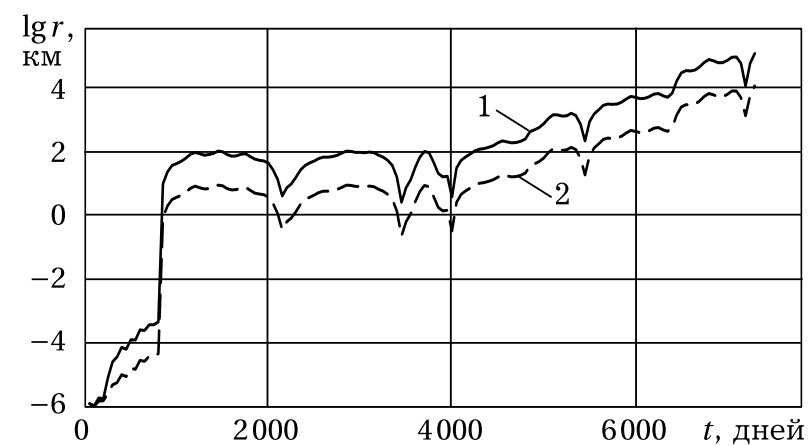

Логарифм отклонения $r$ (км) при шаге 0,2 и 0,125 дня в зависимости от интервала интегрирования $t$ (дней) при использовании в моменты тесных сближений: 1 -метода Эверхарта; 2-метода Адамса

является применение метода Адамса-Мултона тсутствии. А метод Эверхарта следует использовать только для закладки начальных значений многошагового метода.

Работа выполнена при ббнансовой поддержке Федералъного агентства по образованию (проект РНП. 2.1.1.1689)

\section{БИБЛИОГРАФИЧЕСКИЙ СПИСОК}

1. Холл, Дж. Современные численные методы решения обыкновенных дифференциальных уравнений [Текст] / Дж. Холл, Дж. Уатт. - М.: Мир, 1979. - 312 с.

2. Абрамов, В.В. Применение методов Адамса к решению уравнений движения больших планет, Луны и Солнца [Текст] / В.В. Абрамов / Мат. моделирование и краевые задачи: Тр. Третьей Всерос. научн. конф. - Самара: СамГТУ, 2006. - Ч. 3. - С. 13-19. - ISBN 5--7964--0802--X.

3. Абрамов В.В. Эффрективность метода Адамса-Мултона при математическом моделировании движения малых тел Солнечной системы [Текст] / В. В. Абрамов / Нелинейный динамический анализ-2007: Тез. докл. междунар. конгр. - СПб.: СПбГУ, 2007. - С. 184.

4. Everhart, E. Implicit single methods for integrating orbits [Text] / E.Everhart // Celestial mechanics. - 1974.No. $10 .-$ P. $35-55$.

5. Заусаев, А.Ф. Применение метода Эверхарта 31-го порядка для решения уравнений движения больших планет [Текст] / А. Ф. Заусаев, А. А. Заусаев, А. Г. Ольхин // Тр. ГАИШ. - 2004. - Т. LXXV: ВАК-2004. - С. $209-210$.

6. Newhall, X.X. DE 102: a numerically integrated ephemeris of the Moon and planets spanning forty-four centuries [Text] / X. X. Newhall, E. M. Standish, Jr. and J. G. Williams // Astron. Astrophys. - 1983. - No. 125. - P. $150-167$.

7. Абрамов, В.В. Моделирование сближений астероида 99942 Арорhis с внутренними планетами и Луной [Текст] / В. В. Абрамов, С. С. Денисов, Л. А. Соловьев / Тез. докл. XXXII Самарской обл. студ. научн. конф. - Самара, 2006. - Ч. 1. - C. 101 .

8. Абрамов, В.В. Математическое моделирование движения малых тел Солнечной системы на основе методов Адамса [Текст] / В. В. Абрамов // Вестн. Сам. госуд. техн. ун-та. Сер.: Физ.-мат. науки. $-2006 .-$ № 43. - С. 192194. - ISBN 5-7964-0877-1. 